\title{
STABILITY AND LINEAR INDEPENDENCE ASSOCIATED WITH WAVELET DECOMPOSITIONS
}

\author{
Rong-Qing Jia* \\ Department of Mathematics \\ University of Oregon \\ Eugene, OR 97403 \\ U.S.A.
}

\author{
Jianzhong Wang \\ Department of Mathematics \\ Wuhan University \\ Wuhan, Hubei 430072 \\ P. R. China
}

\begin{abstract}
Wavelet decompositions are based on basis functions satisfying refinement equations. The stability, linear independence and orthogonality of the integer translates of basis functions play an essential role in the study of wavelets. In this paper we characterize these properties in terms of the mask sequence in the refinement equation satisfied by the basis function.
\end{abstract}

AMS Subject Classifications: 42 C 05, 41 A 30

Key Words and Phrases: wavelets, wavelet decompositions, refinement equations, stability, linear independence, orthogonality

*Present Address: Department of Mathematics, University of Alberta, Edmonton, Canada T6G 2G1 


\section{Introduction}

The purpose of this paper is to investigate the stability, linear independence and orthogonality of the integer translates of a refinable function. These concepts play an essential role in the study of wavelet decompositions.

A locally integrable function $\phi$ is said to be refinable if it satisfies a refinement equation

$$
\phi=\sum_{j \in \mathbb{Z}} b(j) \phi(2 \cdot-j)
$$

where $b: \mathbb{Z} \rightarrow \mathbb{C}$ is a sequence and is called the mask for the refinement equation (1). Throughout this paper we assume that $\phi$ is compactly supported and $b$ is supported on a finite set, i.e., $b(j)=0$ except for finitely many $j \in \mathbb{Z}$. The Fourier-Laplace transform of $\phi$ is defined to be

$$
\hat{\phi}(\zeta):=\int_{\mathbb{R}} \phi(x) e^{-i x \zeta} d x \quad(\zeta \in \mathbb{C})
$$

Here and throughout this paper $i$ denotes the imaginary unit $\sqrt{-1}$. Restricted to $\mathbb{R}, \hat{\phi}$ becomes the Fourier transform of $\phi$.

As usual, for $1 \leq p \leq \infty$, we denote by $L^{p}(\mathbb{R})$ the Banach space of all complex-valued functions $f$ for which

$$
\|f\|_{p}:=\left(\int_{\mathbb{R}}|f(x)|^{p} d x\right)^{1 / p}<\infty
$$

We denote by $L_{c}^{p}(\mathbb{R})$ the subspace consisting of all compactly supported functions in $L^{p}(\mathbb{R})$. Analogously, let $\ell^{p}(\mathbb{Z})(1 \leq p \leq \infty)$ be the Banach space of all complex-valued sequences $a=(a(j))_{j \in \mathbb{Z}}$ for which

$$
\|a\|_{p}:=\left(\sum_{j \in \mathbb{Z}}|a(j)|^{p}\right)^{1 / p}<\infty
$$

We observe that $L^{2}(\mathbb{R})$ is a Hilbert space with the inner product given by

$$
\langle f, g\rangle:=\int_{\mathbb{R}} f(x) \overline{g(x)} d x
$$

Let now $\phi$ be a function in $L_{c}^{2}(\mathbb{R})$ satisfying the refinement equation (1). If $\phi$ has orthonormal integer translates, i.e.,

$$
\langle\phi(\cdot-m), \phi(\cdot-n)\rangle=\delta_{m n},
$$


where $\delta_{m n}$ is the Kronecker symbol, then with

$$
\psi:=\sum_{j \in \mathbb{Z}}(-1)^{j} \overline{b(1-j)} \phi(2 \cdot-j)
$$

the collection $\left\{\sqrt{2^{j}} \psi\left(2^{j} \cdot-k\right): j, k \in \mathbb{Z}\right\}$ forms an orthonormal basis of wavelets for $L^{2}(\mathbb{R})$ (see [13]).

It is well known that a function $\phi \in L_{c}^{2}(\mathbb{R})$ has orthonormal integer translates if and only if

$$
\sum_{k \in \mathbb{Z}}|\hat{\phi}(\xi+2 \pi k)|^{2}=1 \quad \text { for all } \xi \in \mathbb{R}
$$

If $\phi$ further satisfies the refinement equation (1), then it is desirable to characterize the orthogonality of the integer translates of $\phi$ in terms of the mask $b$. To this end, we take Fourier-Laplace transforms of both sides of (1) and obtain

$$
\hat{\phi}(\zeta)=\hat{\phi}(\zeta / 2) \tilde{b}\left(e^{-i \zeta / 2}\right) / 2 \text { for all } \zeta \in \mathbb{C}
$$

where $\tilde{b}(z)$ is the symbol of $b$ :

$$
\tilde{b}(z):=\sum_{j \in \mathbb{Z}} b(j) z^{j}, \quad z \in \mathbb{C} \backslash\{0\}
$$

Let

$$
H(\zeta):=\sum_{j \in \mathbb{Z}} b(j) e^{-i j \zeta} / 2=\tilde{b}\left(e^{-i \zeta}\right) / 2, \quad \zeta \in \mathbb{C} .
$$

Then $H$ is a $2 \pi$-periodic function. It was shown in [13], [7] and [12] that the following conditions are necessary for $\phi$ to have orthogonal integer translates:

$$
\begin{gathered}
H(0)=1 \\
|H(\omega)|^{2}+|H(\omega+\pi)|^{2}=1 \quad \text { for all } \omega \in \mathbb{R} .
\end{gathered}
$$

However, these conditions are not sufficient. A counterexample was provided in [7] and [12]. Let $\phi$ be the characteristic function of the interval $[0,3)$. Then $\phi$ satisfies the refinement equation (1) with $H(\omega)=\left(1+e^{-i 3 \omega}\right) / 2(\omega \in \mathbb{R})$, which meets the conditions (5) and (6). But $\phi$ is not orthogonal to $\phi(\cdot-1)$.

A question naturally arises: Under what condition on $H$ in addition to (5) and (6) does $\phi$ have orthonormal integer translates? This problem has been investigated by Daubechies 
[7][8], Mallat [12] and Cohen [3][4]. In particular, Cohen[3] gave a condition on $H$ which is both necessary and sufficient for $\phi$ to have orthogonal integer translates. His result is related to the concept of stability. Given a function $\phi \in L_{c}^{2}(\mathbb{R})$, we say that the integer translates $\phi(\cdot-j)(j \in \mathbb{Z})$ are stable, if there exist two positive constants $A$ and $B$ such that for any sequence $a \in \ell^{2}(\mathbb{Z})$,

$$
A\|a\|_{2} \leq\left\|\sum_{j \in \mathbb{Z}} a(j) \phi(\cdot-j)\right\|_{2} \leq B\|a\|_{2}
$$

In other words, the integer translates of $\phi$ are stable if the collection $\{\phi(\cdot-j): j \in \mathbb{Z}\}$ is an unconditional basis for the subspace of $L^{2}(\mathbb{R})$ generated by them. Cohen's result can be interpreted as follows: A refinable function $\phi$ with $\hat{\phi}(0)=1$ has orthonormal integer translates if and only if $\phi$ has stable integer translates and the corresponding function $H$ satisfies the condition (6). Thus the problem reduces to characterizing the stability of the integer translates of a refinable function in terms of its mask.

Stability itself plays an important role in multiresolution analysis as introduced by Mallat [12]. Given a refinable function $\phi \in L_{c}^{2}(\mathbb{R})$, let

$$
V_{j}:=\left\{\sum_{k \in \mathbb{Z}} a(k) \phi\left(2^{j} \cdot-k\right): a \in \ell^{2}(\mathbb{Z})\right\}, \quad j \in \mathbb{Z} .
$$

Then the sequence $\left(V_{j}\right)_{j \in \mathbb{Z}}$ forms a multiresolution approximation of $L^{2}(\mathbb{R})$ if and only if $\phi$ has stable integer translates. This result has been extended by Jia and Micchelli [10] to functions in several variables.

Linear independence is a concept closely related to stability. This concept is useful in constructing wavelet decompositions by some algebraic tools (see [14] and [10]). In the next section we shall demonstrate that orthogonality implies linear independence, and linear independence implies stability.

The main results of this paper are the characterizations of the stability, linear independence and orthogonality of the integer translates of a refinable function in terms of the zero distribution of the symbol of the mask. We shall also give several examples to illustrate these results.

\section{Stability and Linear Independence}

In this section, we study the stability and linear independence of a compactly supported distribution in terms of its Fourier-Laplace transform. 
We may generalize the concept of stability as follows. Let $\phi \in L_{c}^{p}(\mathbb{R})(1 \leq p \leq \infty)$. We say that the integer translates $\phi(\cdot-j)(j \in \mathbb{Z})$ are $\ell^{p}$-stable, if there exist positive constants $A_{p}$ and $B_{p}$ such that for any sequence $a \in \ell^{p}(\mathbb{Z})$,

$$
A_{p}\|a\|_{p} \leq\left\|\sum_{j \in \mathbb{Z}} a(j) \phi(\cdot-j)\right\|_{p} \leq B_{p}\|a\|_{p}
$$

In other words, the integer translates of $\phi$ are $\ell^{p}$-stable if the collection $\{\phi(\cdot-j): j \in \mathbb{Z}\}$ is an unconditional basis for the subspace of $L^{p}(\mathbb{R})$ generated by them. Note that the second inequality in (8) automatically holds for $\phi \in L_{c}^{p}(\mathbb{R})$; hence only the first inequality in (8) is significant. It is well known that $\phi \in L_{c}^{2}(\mathbb{R})$ satisfies $(7)$ if and only if

$$
(\hat{\phi}(\xi+2 \pi k))_{k \in \mathbb{Z}} \neq 0 \quad \text { for any } \xi \in \mathbb{R}
$$

where 0 means the zero sequence on $\mathbb{Z}$. It is less known that (9) is also equivalent to the $\ell^{p}$-stability of the integer translates of $\phi \in L_{c}^{p}(\mathbb{R})$ for any $p, 1 \leq p \leq \infty$. For this, see [9] and $[10]$.

We observe that the condition (9) does not depend on $p$. Moreover, (9) does not require that $\phi \in L_{c}^{p}(\mathbb{R})$. Indeed, if $\phi$ is a compactly supported distribution, then its Fourier-Laplace transform $\hat{\phi}$ is an entire function on $\mathbb{C}$. Thus we may say that a compactly supported distribution $\phi$ has stable integer translates if its Fourier transform $\hat{\phi}$ satisfies the condition (9).

To introduce the concept of linear independence, we denote by $\ell(\mathbb{Z})$ the linear space of all complex-valued sequences on $\mathbb{Z}$, and consider the subspace $N(\phi)$ of $\ell(\mathbb{Z})$ given by

$$
N(\phi):=\left\{a \in \ell(\mathbb{Z}): \sum_{j \in \mathbb{Z}} a(j) \phi(\cdot-j)=0\right\} .
$$

If $N(\phi)$ is trivial, i.e., $N(\phi)=\{0\}$, then we say that the integer translates of $\phi$ are linearly independent. Let

$$
K(\phi):=\left\{z \in \mathbb{C} \backslash\{0\}:\left(z^{j}\right)_{j \in \mathbb{Z}} \in N(\phi)\right\} .
$$

When $\phi$ is a compactly supported continuous function, Dahmen and Micchelli [5] showed that $N(\phi)$ is trivial if and only if $K(\phi)$ is empty. Ron [15] extended their result to the case in which $\phi$ could be a compactly supported distribution and observed that $e^{i \zeta} \in K(\phi)$ if and only if $(\hat{\phi}(\zeta+2 \pi k))_{k \in \mathbb{Z}}=0$. Thus their result can be stated as follows: The integer translates of $\phi$ are linearly independent if and only if

$$
(\hat{\phi}(\zeta+2 \pi k))_{k \in \mathbb{Z}} \neq 0 \quad \text { for any } \zeta \in \mathbb{C} .
$$


Note that (11) is equivalent to $K(\phi)=\emptyset$, while (9) is equivalent to $K(\phi) \cap T=\emptyset$, where $T$ is the unit circle $\{z \in \mathbb{C}:|z|=1\}$. Since $\phi$ is a distribution on $\mathbb{R}$, we deduce from $[15$, Proposition 2.1] that there is a Laurent polynomial $p$ on $\mathbb{C}$ such that

$$
K(\phi)=\{z \in \mathbb{C} \backslash\{0\}: p(z)=0\} .
$$

The Laurent polynomial $p$ is nonzero as long as $\phi$ is not identically zero. This shows that $K(\phi)$ is a finite set unless $\phi$ is identically zero.

Comparing (11) with (9), we see that linear independence implies stability. Moreover, if $\phi \in L_{c}^{2}(\mathbb{R})$ has orthonormal integer translates, then these integer translates are linearly independent. To see this, let $a$ be a sequence on $\mathbb{Z}$ such that $f:=\sum_{j \in \mathbb{Z}} a(j) \phi(\cdot-j)=0$. Then by the orthonormality, we have

$$
a(j)=\langle f, \phi(\cdot-j)\rangle=0 \quad \text { for all } j \in \mathbb{Z} \text {. }
$$

The function $\phi$ given below is an example of a refinable function whose integer translates are linearly independent but not orthogonal:

$$
\phi(x)= \begin{cases}1+x, & \text { if }-1 \leq x \leq 0 \\ 1-x, & \text { if } 0<x \leq 1 \\ 0, & \text { elsewhere }\end{cases}
$$

Admittedly, the inequality (8) does not make any sense when $\phi$ is not a function in $L^{p}(\mathbb{R})$. However, it is convenient and useful to consider distribution solutions to the refinement equation (1). For example, as was pointed out by Strang [16], the Dirac measure $\delta$ satisfies the refinement equation (1) with the mask $b$ given by $b(j)=2 \delta_{0 j}$. In general, Daubechies [8] proved that if $\sum_{j \in \mathbb{Z}} b(j)=2$, i.e., $H(0)=1$, then the infinite product

$$
\prod_{k=1}^{\infty} H\left(\zeta / 2^{k}\right)
$$

converges uniformly on compact sets to an entire function $f(\zeta)$ on $\mathbb{C}$ for which there exist positive constants $A, C$ and an integer $N \geq 0$ such that

$$
|f(\zeta)| \leq C(1+|\zeta|)^{N} e^{A|\operatorname{Im} \zeta|} \text { for all } \zeta \in \mathbb{C} .
$$

Then by the Paley-Wiener-Schwartz theorem, $f$ is the Fourier-Laplace transform of some compactly supported distribution $\phi_{1}$. From the construction of $\phi_{1}$, we see that $\hat{\phi}_{1}(0)=1$ and

$$
\hat{\phi}_{1}(\zeta)=H(\zeta / 2) \hat{\phi}_{1}(\zeta / 2) \quad(\zeta \in \mathbb{C}) .
$$


Therefore $\phi_{1}$ satisfies the refinement equation (1). Moreover, any solution $\phi$ of (1) satisfies

$$
\hat{\phi}(\zeta)=\hat{\phi}(0) \hat{\phi}_{1}(\zeta)=\hat{\phi}(0) \prod_{k=1}^{\infty} H\left(\zeta / 2^{k}\right) \quad(\zeta \in \mathbb{C})
$$

In particular, $\hat{\phi}(0) \neq 0$ unless $\hat{\phi}$ is identically zero.

\section{Main Results}

In this section we state our main results and give several examples to illustrate them, leaving the proof to the next section.

To state our results we need to recall Euler's theorem from number theory (e.g., see [11, Theorem 3.10]). Euler's theorem says that if $q$ and $m$ are relatively prime integers, $m>1$, then

$$
q^{\varphi(m)} \equiv 1(\bmod m)
$$

where $\varphi$ is the Euler function, i.e., $\varphi(m)$ is the number of positive integers $\leq m$ which are relatively prime to $m$. Let $h$ be the smallest positive integer such that

$$
q^{h} \equiv 1(\bmod m)
$$

Then $h$ is called the order of $q(\bmod m)$, and is denoted by $\operatorname{ord}_{m} q$. It is well known that $\operatorname{ord}_{m} q$ divides $\varphi(m)$.

The following three theorems establish criteria for a refinable distribution to have stable, linearly independent, or orthogonal integer translates. In these theorems we assume that $b$ is a finitely supported sequence on $\mathbb{Z}$ with $\sum_{j \in \mathbb{Z}} b(j)=2, \tilde{b}(z)$ is the symbol of $b$ as given in (3), and $\phi$ is the distribution solution to the refinement equation (1) with $\hat{\phi}(0)=1$.

Theorem 1. The integer translates of $\phi$ are stable if and only if the symbol $\tilde{b}(z)$ satisfies the following two conditions:

$\left(1^{\circ}\right) \tilde{b}(z)$ does not have any symmetric zeros on the unit circle $T$;

$\left(2^{\circ}\right)$ For any odd integer $m>1$ and a primitive $m$ th root $\omega$ of unity, there exists an integer $d, 0 \leq d<\operatorname{ord}_{m} 2$, such that $\tilde{b}\left(-\omega^{2^{d}}\right) \neq 0$.

Remark 1. With $h:=\operatorname{ord}_{m} 2$ we have $2^{h} \equiv 1(\bmod m)$, and so $\omega^{2^{h}}=\omega$. Thus the condition $\left(2^{\circ}\right)$ can be restated as follows: There exists an integer $d \geq 0$ such that $\tilde{b}\left(-\omega^{2^{d}}\right) \neq 0$. 
Remark 2. The conditions $\left(1^{\circ}\right)$ and $\left(2^{\circ}\right)$ are easy to check if one knows the zero distribution of the symbol $\tilde{b}(z)$. Usually, the zeros of $\tilde{b}(z)$ are preassigned.

Theorem 2. The integer translates of $\phi$ are linearly independent if and only if the symbol $\tilde{b}(z)$ satisfies the condition $\left(2^{\circ}\right)$ of Theorem 1 and the following condition: $\tilde{b}(z)$ does not have any symmetric zeros in $\mathbb{C} \backslash\{0\}$.

Theorem 3. The distribution $\phi$ is in $L_{c}^{2}(\mathbb{R})$ and has orthogonal integer translates if and only if the symbol $\tilde{b}(z)$ satisfies the condition $\left(2^{\circ}\right)$ of Theorem 1 and the following condition:

$$
|\tilde{b}(z)|^{2}+|\tilde{b}(-z)|^{2}=4 \quad \text { for all } z \in T \text {. }
$$

Remark 3. The condition (12) is equivalent to (6).

Before proving these theorems, we give several examples to illustrate them.

Example 1. Let $\phi_{n}:=\chi_{[0, n)} / n$, where $n$ is a positive integer and $\chi_{[0, n)}$ is the characteristic function of the interval $[0, n)$. Then $\phi_{n}$ satisfies the refinement equation $(1)$ with $\tilde{b}(z)=$ $1+z^{n}$. If $n$ is an even integer, then $\tilde{b}$ has symmetric zeros on the unit circle. If $n$ is an odd integer bigger than 1 , then with $\omega=e^{i 2 \pi / n}, \tilde{b}\left(-\omega^{m}\right)=0$ for all integers $m$. This shows that the integer translates of $\phi_{n}$ are not stable except for the case $n=1$. Note that when $n$ is odd, the symbol $\tilde{b}(z)$ satisfies the condition (12).

Example 2. (This example also appeared in [4, Chap. 2, Corollary 2.1], where a different argument was used.) Let $H$ be defined as in (4). It was proved by Mallat [12, Theorem 2] that $\phi$ has orthogonal integer translates provided that $H$ satisfies (5),(6) and the following condition:

$$
H(\xi) \neq 0 \quad \text { for } \xi \in[-\pi / 2, \pi / 2] .
$$

Under the restriction that $b$ is finitely supported, we can strengthen Mallat's result as follows: If $H$ satisfies (5) and (6), and if

$$
H(\xi) \neq 0 \quad \text { for } \xi \in[-\pi / 3, \pi / 3]
$$

then $\phi$ has orthogonal integer translates. To see this, we let $\omega=e^{-i \xi}$ be a primitive $m$ th root of unity for some odd integer $m>1$. If $2 \pi / 3 \leq|\xi| \leq 4 \pi / 3$, then either $\xi-\pi$ or $\xi+\pi$ lies in the interval $[-\pi / 3, \pi / 3]$, hence

$$
\tilde{b}(-\omega)=2 H(\xi-\pi)=2 H(\xi+\pi) \neq 0
$$


by the assumption. If $|\xi| \leq 2 \pi / 3$, then $2 \pi / 3 \leq 2^{d}|\xi| \leq 4 \pi / 3$ for some integer $d>0$; hence $\tilde{b}\left(-\omega^{2^{d}}\right) \neq 0$. Therefore, by Theorem 3 , $\phi$ has orthogonal integer translates.

Example 3. Let $b$ be the sequence given by its symbol

$$
\tilde{b}(z)=2\left(\frac{1+z}{2}\right)^{3} \frac{1+2 z^{2}}{3} \text {. }
$$

Let $\phi$ be the unique solution to (1) with the mask $b$ as given above satisfying $\hat{\phi}(0)=1$. Then $\phi$ actually is a continuous function. To see this, we observe that for $\xi \in \mathbb{R}$,

$$
|\hat{\phi}(\xi)|=\left|\prod_{k=1}^{\infty}\left[\tilde{b}\left(e^{-i \xi / 2^{k}}\right) / 2\right]\right| \leq|\sin (\xi / 2) /(\xi / 2)|^{3} .
$$

Thus the function given by $\xi \mapsto \xi \hat{\phi}(\xi)(\xi \in \mathbb{R})$ is in $L^{1}(\mathbb{R})$, hence the derivative $\phi^{\prime}$ is in $L^{\infty}(\mathbb{R})$. This shows that $\phi$ is continuous. Moreover, by Theorem $1, \phi$ has stable integer translates. But, by Theorem 2, the integer translates of $\phi$ are linearly dependent, since $\tilde{b}(z)$ has symmetric zeros $\pm i / \sqrt{2}$.

\section{Proof of the Main Results}

To prove Theorem 1 we need a lemma. Its proof employs [1, Lemma 6.6].

Lemma 1. Let $\phi$ be a nonzero compactly supported distribution satisfying the refinement equation (1) with $\sum_{j \in \mathbb{Z}} b(j)=2$, and let $K(\phi)$ be as given in (10). Suppose that $\tilde{b}(z)$ does not have any symmetric zeros in $\mathbb{C} \backslash\{0\}$ (resp. on the unit circle $T$ ). Then $z \in K(\phi)$ (resp. $z \in K(\phi) \cap T$ ) only if $z$ is a primitive $m$ th root of unity for some odd integer $m>1$.

Proof: Pick $z_{0}=e^{i \zeta_{0}} \in K(\phi)$. Then

$$
\hat{\phi}\left(\zeta_{0}+2 k \pi\right)=0 \quad \text { for all } k \in \mathbb{Z} \text {. }
$$

In particular, $z_{0} \neq 1$, because $\hat{\phi}(0) \neq 0$. By $(2)$ we have that for all $k \in \mathbb{Z}$,

$$
0=\hat{\phi}\left(\zeta_{0}+4 k \pi\right)=\hat{\phi}\left(\zeta_{0} / 2+2 k \pi\right) \tilde{b}\left(e^{-i \zeta_{0} / 2}\right) / 2
$$

and

$$
0=\hat{\phi}\left(\zeta_{0}+2 \pi+4 k \pi\right)=\hat{\phi}\left(\zeta_{0} / 2+\pi+2 k \pi\right) \tilde{b}\left(-e^{-i \zeta_{0} / 2}\right) / 2 .
$$

By the hypothesis, $\tilde{b}(z)$ does not have any symmetric zeros in $\mathbb{C} \backslash\{0\}$; hence at least one of $\tilde{b}\left(e^{-i \zeta_{0} / 2}\right)$ and $\tilde{b}\left(-e^{-i \zeta_{0} / 2}\right)$ is nonzero. If $z_{0}=e^{i \zeta_{0}} \in T$, then $\zeta_{0} \in \mathbb{R}$, so the above 
argument is valid under the weaker condition that $\tilde{b}(z)$ does not have any symmetric zeros on the unit circle $T$. This observation together with (13) and (14) tells us that either

$$
\left(\hat{\phi}\left(\zeta_{0} / 2+2 k \pi\right)\right)_{k \in \mathbb{Z}}=0
$$

or

$$
\left(\hat{\phi}\left(\zeta_{0} / 2+\pi+2 k \pi\right)\right)_{k \in \mathbb{Z}}=0 .
$$

In other words, either $e^{i \zeta_{0} / 2}$ or $e^{i\left(\zeta_{0} / 2+\pi\right)}$ is in $K(\phi)$. This shows that there exists $z_{1} \in K(\phi)$ such that $z_{1}^{2}=z_{0}$. Repeating this process, we can find a sequence $\left(z_{n}\right)_{n=0,1, \ldots}$ in $K(\phi)$ such that $z_{n}^{2}=z_{n-1}(n=1,2, \ldots)$. But $K(\phi)$ is a finite set. Hence there must be integers $r, s, 0 \leq r<s$ such that $z_{r}=z_{s}$. Write $\omega=z_{s}$. Then

$$
\omega^{2^{s-r}}=z_{r}=z_{s}=\omega
$$

Note that $\omega \neq 1$, for otherwise $z_{0}=\omega^{2^{s}}$ would equal 1. From the above equation on $\omega$ we conclude that $\omega$ is a primitive $m$ th root of unity for some odd integer $m>1$, and therefore so is $z_{0}=\omega^{2^{s}}$.

On the basis of Lemma 1, Theorem 2 can be easily derived from Theorem 1 . To see this, let $\phi$ satisfy the conditions of Theorem 2 . Then the symbol $\tilde{b}(z)$ does not have any symmetric zeros in $\mathbb{C} \backslash\{0\}$, hence $K(\phi)$ is a subset of the unit circle $T$ by Lemma 1 . But Theorem 1 tells us that $K(\phi) \cap T$ is empty, therefore $K(\phi)$ is empty; that is, $\phi$ has linearly independent integer translates. Moreover, Theorem 3 also follows immediately from Theorem 1. Indeed, if $\phi$ satisfies the conditions of Theorem 3, then it satisfies those of Theorem 1, because (12) implies the condition $\left(1^{\circ}\right)$ of Theorem 1 . Thus $\phi$ has stable integer translates, and because of (12) and the fact that $\hat{\phi}(0)=1$ it has orthonormal integer translates. We are now in a position to prove Theorem 1.

Proof of Theorem 1: $\quad$ First, it was proved in [2, Theorem 2.1] that the condition $\left(1^{\circ}\right)$ is necessary. For reader's convenience, we include the proof here. Suppose that $\tilde{b}(z)$ has symmetric zeros on the unit circle, say

$$
\tilde{b}\left(e^{-i \omega}\right)=\tilde{b}\left(-e^{-i \omega}\right)=0
$$

for some $\omega \in \mathbb{R}$. Then for any integer $k$ we have

$$
\hat{\phi}(2 \omega+4 k \pi)=\hat{\phi}(\omega+2 k \pi) \tilde{b}\left(e^{-i \omega}\right) / 2=0
$$


and

$$
\hat{\phi}(2 \omega+(4 k+2) \pi)=\hat{\phi}(\omega+(2 k+1) \pi) \tilde{b}\left(-e^{-i \omega}\right) / 2=0 .
$$

This shows that the integer translates of $\phi$ are not stable.

Second, we show that the condition $\left(2^{\circ}\right)$ is also necessary for $\phi$ to have stable integer translates. Suppose to the contrary that for some odd integer $m>1$ there exists a primitive $m$ th root $\omega$ such that

$$
\tilde{b}\left(-\omega^{2^{d}}\right)=0 \quad \text { for all integers } d \geq 0 .
$$

Then $\omega$ has the form $e^{-i 2 n \pi / m}$, where $n$ is an integer relatively prime to $m$. We claim that for all integers $k$

$$
\hat{\phi}(2 n \pi / m+2 k \pi)=0 .
$$

To prove (15), we pick an integer $k$ and write $n+k m$ in the form $2^{p} q$, where $p$ is a nonnegative integer and $q$ is an odd integer. We observe from (2) that

$$
\hat{\phi}(2 n \pi / m+2 k \pi)=\hat{\phi}\left(2^{p+1} q \pi / m\right)=\hat{\phi}(q \pi / m) \prod_{j=0}^{p}\left[\tilde{b}\left(e^{-i 2^{j} q \pi / m}\right) / 2\right] .
$$

Hence in order to prove (15) it suffices to show that $\tilde{b}\left(e^{-i q \pi / m}\right)=0$. For this purpose, we invoke Euler's theorem to find an integer $r>p$ such that $2^{r} \equiv 1(\bmod m)$. It follows that

$$
q \equiv 2^{r} q \equiv 2^{r-p}\left(2^{p} q\right) \equiv 2^{r-p} n(\bmod m)
$$

In other words, $q-2^{r-p} n=m \ell$ for some integer $\ell$. The integer $\ell$ must be odd, for $q$ is odd, while $2^{r-p} n$ is even. From this we see that

$$
\tilde{b}\left(e^{-i q \pi / m}\right)=\tilde{b}\left(-e^{-i 2^{r-p} n \pi / m}\right)=\tilde{b}\left(-\omega^{2^{d}}\right)=0,
$$

where $d=r-p-1$. This completes the proof of the necessity part.

Third, we prove that the conditions $\left(1^{\circ}\right)$ and $\left(2^{\circ}\right)$ are sufficient for $\phi$ to have stable integer translates. We claim that if $\tilde{b}(z)$ does not have any symmetric zeros on the unit circle $T$, and if $m$ and $n$ are two relatively prime integers with $m>1$ being odd, then

$$
e^{i 4 \pi n / m} \in K(\phi) \Longrightarrow e^{i 2 \pi n / m} \in K(\phi) \text { and } \tilde{b}\left(-e^{-i 2 \pi n / m}\right)=0 .
$$

To this end, we observe that $e^{i(2 \pi n / m+\pi)}$ is not an odd root of unity, hence by Lemma 1 it does not belong to $K(\phi)$. This shows that there exists an integer $k$ such that

$$
\hat{\phi}(2 \pi n / m+\pi+2 k \pi) \neq 0 \text {. }
$$


Since $e^{i 4 \pi n / m} \in K(\phi)$, we have

$$
\hat{\phi}(4 \pi n / m+2 j \pi)=0 \text { for all } j \in \mathbb{Z} .
$$

By (2) we have

$$
\hat{\phi}(4 \pi n / m+(4 k+2) \pi)=\hat{\phi}(2 \pi n / m+\pi+2 k \pi) \tilde{b}\left(-e^{-i 2 \pi n / m}\right) / 2 .
$$

This together with (17) and (18) yields

$$
\tilde{b}\left(-e^{-i 2 \pi n / m}\right)=0 .
$$

But $\tilde{b}(z)$ does not have any symmetric zeros on the unit circle, hence it follows that

$$
\tilde{b}\left(e^{-i 2 \pi n / m}\right) \neq 0 \text {. }
$$

Invoking (2) again, we see that for all $j \in \mathbb{Z}$,

$$
0=\hat{\phi}(4 \pi n / m+4 j \pi)=\hat{\phi}(2 \pi n / m+2 j \pi) \tilde{b}\left(e^{-i 2 \pi n / m}\right) / 2 .
$$

It follows that for all integers $j$,

$$
\hat{\phi}(2 \pi n / m+2 j \pi)=0,
$$

which shows that $e^{i 2 \pi n / m} \in K(\phi)$. Thus the claim (16) has been proved.

Let us now finish the proof of the sufficiency part. It suffices to prove that $K(\phi) \cap T$ is the empty set. If $K(\phi) \cap T$ were nonempty, then by Lemma 1, it would contain a primitive odd root of unity, say $e^{i 2^{p} n \pi / m}$, where $m$ and $n$ are two relatively prime odd integers with $m>1$ and $p$ is a positive integer. Let $\omega:=e^{-i 2 n \pi / m}$. Then $\omega$ is a primitive $m$ th root of unity. With $h:=\operatorname{ord}_{m} 2$, we have $2^{h} \equiv 1(\bmod m)$. Hence

$$
e^{i 2^{p+h} n \pi / m}=e^{i 2^{p} n \pi / m} \in K(\phi) .
$$

Applying (16) to $e^{i 2^{p+h} n \pi / m}$ repeatedly, we see that for $0 \leq d<h$,

$$
\tilde{b}\left(-\omega^{2^{d}}\right)=\tilde{b}\left(-e^{-i 2^{d+1} n \pi / m}\right)=0,
$$

which contradicts the condition $\left(2^{\circ}\right)$. This shows that $K(\phi) \cap T$ is empty, as desired.

Final Remark. After this paper was submitted, we became aware that A. Cohen had established a criterion for a refinable function to have orthonormal integer translates in his thesis [4, Chap. 2, Theorem 2.2]. His result is very similar to Theorem 3 of this paper. In this paper, however, we also characterized the stability and linear independence of the integer translates of a refinable function, and clarified the relationship among stability, linear independence and orthogonality. 


\section{References}

[1] A. S. Cavaretta, W. Dahmen, and C. A. Micchelli, Stationary Subdivision, Memoir of Amer. Math. Soc., No. 453 (1991).

[2] C. K. Chui and J. Z. Wang, A general framework of compactly supported splines and wavelets, CAT Report 219, Texas A\&M University (1990).

[3] A. Cohen, Ondelettes, analysis multirésolutions et filtres mirroirs en quadrature, Ann. Inst. H. Poincaré, 7 (1990), 439-459.

[4] A. Cohen, Ondelettes, analysis multiresolutions et traitement numerique du signal, Ph.D. Thesis, 1990, Université de Paris IX (Dauphine), France.

[5] W. Dahmen and C. A. Micchelli, Translates of multivariate splines, Linear Algebra and its Applications, 52/53 (1983), 217-234.

[6] W. Dahmen and C. A. Micchelli, On stationary subdivision and the construction of compactly supported orthonormal wavelets, in Multivariate Approximation and Interpolation, W. Haussmann and K. Jetter (eds.), Birkhäuser Verlag, Basel (1990), pp. 69-89.

[7] I. Daubechies, Orthonormal bases of compactly supported wavelets, Comm. Pure and Appl. Math., 41 (1988), 909-996.

[8] I. Daubechies, Ten Lectures on Wavelets, CBMS/NSF Regional Conference Series in Applied Mathematics 61, SIAM, Philadelphia (1992).

[9] R. Q. Jia and C. A. Micchelli, On linear independence of integer translates of a finite number of functions, Proceedings of the Edinburgh Mathematical Society, to appear.

[10] R. Q. Jia and C. A. Micchelli, Using the refinement equation for the construction of pre-wavelets II: Powers of two, in Curves and Surfaces, P. J. Laurent, A. Le Méhauté and L. L. Schumaker (eds.), Academic Press, New York (1991), pp. 209-246.

[11] W. J. LeVeque, Fundamentals of Number Theory, Addison-Wesley Publishing Company, Reading, Massachusetts (1977).

[12] S. G. Mallat, Multiresolution approximations and wavelet orthonormal basis of $L^{2}(\mathbb{R})$, Trans. Amer. Math. Soc., 315 (1989), 69-87.

[13] Y. Meyer, Ondelettes et fonctions splines, Seminaire Equations aux Derivees Partielles, Ecole Polytechnique, Paris, France (1986).

[14] C. A. Micchelli, Using the refinement equation for the construction of pre-wavelets, Numerical Algorithms, 1 (1991), 75-116.

[15] A. Ron, A necessary and sufficient condition for the linear independence of the integer translates of a compactly supported distribution, Constr. Approx., 5 (1989), 297-308. 
[16] G. Strang, Wavelets and dilation equations: A brief introduction, SIAM Review, 31 (1989), 614-627. 